Virginia Commonwealth University

VCU Scholars Compass

\title{
Focused attention, open monitoring and automatic self-transcending: Categories to organize meditations from Vedic, Buddhist and Chinese traditions
}

Fred Travis

Maharishi University of Management

Jonathan Shear

Virginia Commonwealth University, jshear@vcu.edu

Follow this and additional works at: http://scholarscompass.vcu.edu/phil_pubs

Part of the Philosophy Commons

Copyright $(\odot$ Elsevier Ltd. NOTICE: this is the author's version of a work that was accepted for publication in Consciousness and Cognition. Changes resulting from the publishing process, such as peer review, editing, corrections, structural formatting, and other quality control mechanisms may not be reflected in this document. Changes may have been made to this work since it was submitted for publication. A definitive version was subsequently published in Consciousness and Cognition, Volume 19, Issue 4, December 2010, Pages 1110-1118, doi:10.1016/ j.concog.2010.01.007.

\section{Downloaded from}

http://scholarscompass.vcu.edu/phil_pubs/2

This Article is brought to you for free and open access by the Dept. of Philosophy at VCU Scholars Compass. It has been accepted for inclusion in Philosophy Publications by an authorized administrator of VCU Scholars Compass. For more information, please contact libcompass@vcu.edu. 


\section{Some Reflections on Meditation Research and Consciousness Studies:}

Jonathan Shear, Department of Philosophy

Virginia Commonwealth University

Copyright (C) Journal of Consciousness Studies. This is the author's version of a work that was accepted for publication in the Journal of Consciousness Studies, Vol. 21(3-4), 202-215, 2014. This article may not exactly replicate the final published version.

My interest in meditation and consciousness studies began some fifty years ago when as a Fulbright Scholar in philosophy of science at the London School of Economics I became interested in a remarkable set of experiences described by meditation traditions throughout the world. The experiences themselves were highly unusual. One, the most basic, was reported to be virtually contentless. Others appeared to be of what philosophers might call the "space" of awareness devoid of all the objects such as sensations, images and thoughts that fill our ordinary awareness. These experiences were all often associated with reports of intense happiness, a happiness that seemed to be happiness itself, as contrasted with being happy about something or other. And they all seemed to be produced by a wide variety of very different types of meditation procedures. All of this was remarkable enough. But as a student of philosophy of science, what seemed most remarkable to me was that it was not easy to discount these reports as mere products of people's beliefs and imagination. For it seemed highly unlikely to me that so many traditions would conclude independently that a specific set of highly unusual experiences could be had deep within, accompanied by specific physiological correlates and followed by similar effects, simply on the basis of their divergent, often directly opposed belief systems. There had, I thought, to be an empirical component. The historical record, in other words, seemed a bit like a grand (if unsystematic and uncontrolled) scientific experiment, filtering out the role of belief and suggesting strongly that the experiences, correlates and effects reported had an empirical basis independent of such things as culturally produced beliefs and expectations. As a result it seemed there could be good empirical reasons both to take reports of these unusual experiences seriously, and to see what implications they might have for modern philosophy.

Major developmental psychologists such as Maslow and Kohlberg had by then already been taking these experiences seriously for several years. Maslow, for example, noted that these kinds of experiences appeared to be especially common in the people whom he regarded as most high- 
ly "self-actualized." And Kohlberg held them to be essential for attaining in the highest levels of moral development he described. None of the modern philosophers I had studied however seemed to take these experiences into account at all. So I began to examine whether and where introducing these experiences, in the spirit of new empirical data, into modern Western philosophical discourse might corroborate, falsify or otherwise influence our mind-related philosophical theories. ${ }^{1}$ The result was a series of articles (Shear 1981, 1982, 1983, 1990a, etc.) and a book with chapters on self, ethics, creativity and aspects of Plato's works bringing this work together and examining implications of this approach for Western philosophy as a whole (Shear 1990b, 2014).

I was not alone in this enterprise, of course. Philosophers of various sorts were becoming interested in these experiences. And their reactions varied. One major reaction was to dismiss the notion of core, culture-invariant meditation experiences altogether. Perhaps the most influential of these critics was Steven Katz, who argued from a priori principles that all experiences have to be built up of and shaped by culture-dependent elements such as "images, beliefs, symbols," etc. which "define, in advance" what all of one's experiences will be like. (Katz 1978, pp. $33,59,62$, etc.). As a result the experiences described as "contentless" by so many different traditions have to be different. This argument, however, is likely to appear quite odd to anyone who has actually had an experience that seems to him (or her) to be contentless. For to appear "contentless" an experience would obviously have to appear devoid of all the kinds of content (images, symbols, beliefs, etc.) Katz held necessarily differentiate experiences had in different cultures. Katz's analysis, moreover, flies directly in the face of explicit assertions found the literature of meditation traditions, and emphasized by contemporary teachers, that all such content (including the respective traditions' own canonical content) has to be left behind entirely for the "contentless" experiences to occur. Such straightforward experiential and textual responses are not hard to understand, and after they appeared in print in several venues (Shear 1990c, 1994), Forman 1990), ${ }^{2}$ Katz's opposition to the possibility of culture-invariant meditation experiences

\footnotetext{
${ }^{1}$ To supplement this intellectual work with first-hand experience, I also began some fifty years of daily practice of traditional meditation techniques (five years of Zen, forty-five years of Transcendental Meditation, plus years with techniques from Yoga, years of Qigong) along with martial arts associated with such techniques.

${ }^{2}$ Foreman's volume, with pro and con chapters written by a variety of scholars, was particularly effective in bringing the debate to a wider audience.
} 
faded from view, and the notion that meditation might well be capable of displaying cultureindependent aspects of human awareness gained more traction. ${ }^{3}$

It is now widely accepted that phenomenologically comparable (if not identical) meditation experiences and meditation-related states and stages of growth outside of meditation can often be identified across different traditions and cultures. This view, for example, is often articulated at the biannual Towards a Science of Consciousness conferences, and is prominent at the annual Science and Non-Duality conferences the former conferences gave rise to. It was also what prompted my focus on consciousness studies in the first place. Nevertheless, taken uncritically it can lead to significant problems. For the widespread acceptance of the idea that the same important meditation-related experiences are reported by different meditation systems has often given rise to the simplistic notion that meditation techniques are all essentially the same. Yet in fact meditation systems and their techniques often differ greatly.

Consider for example the following list adapted from a book on major meditation systems with chapters written by people (abbots of monasteries, heads of meditation centers, well-known scholars and teachers, etc.) with expert knowledge of the systems they described:

Zen Buddhism: concentration directed perceptually towards one's breathing, or conceptually towards paradoxes (koans) that defy intellectual resolution.

Taoism: circulation of energy throughout channels of the body.

Transcendental Meditation (TM): relaxed attention to special sounds (mantras) repeated silently within the mind.

Yoga: adds (among its many practices) concentration on energy centers in the body (chakras), the "light" of the mind, and attributes of God.

Theravada Buddhism: dispassionate observation of the impermanence of sensations, thoughts, and whatever else is meditated on, including the self itself.

\footnotetext{
${ }^{3}$ Not long after Forman's book came out, he chaired a panel at an annual meeting of the American Academy of Religion, with Katz and other well known scholars as participants. During the question and answer session after the debate I asked the panelists whether they themselves had ever had the experience they were talking about. The result was very striking: The panelists who argued that experiences could not possibly be the same in different traditions all said that they had never such an experience themselves. The panelists who reported having had such an experience, on the other hand, all argued for the sameness of the experience across traditions. The usefulness of direct experience for understanding the traditional descriptions could hardly be clearer. (Such experience is, of course, not necessary for understanding the logic involved, and one of the panelists who argued for the cultureinvariance of the experience was among those reporting he had never had the experience himself.)
} 
Tibetan Buddhist Tsonghakapa: reasoned deconstruction of the reality of objects experienced in meditation, and practices to quiet the mind.

Integral Yoga: attending to a sense of seeking, and remembering calmness and divinity both during and after meditation.

Kriya yoga: concentration on breath, and on God.

Sufism: following the inner feeling of love for God.

Christian Centering Prayer: repetition of a word of love to stimulate receptiveness to God within. ${ }^{4}$

Thus even a little knowledge about meditation systems in general makes it clear they often differ significantly with regard to

the mental faculties they use (attention, feeling, reasoning, visualization, memory, bodily awareness, etc.), the way these faculties are used (effortlessly, forcefully, actively, passively), and the [purported] objects they are directed to (thoughts, images, concepts, internal energy, breath, subtle aspects of the body, love, God). ${ }^{5}$

Given such differences it would seem reasonable to expect different effects from different meditation techniques on given variables. Techniques that emphasize attention to breathing and those that ignore it, for example, could be expected to have different effects on respiratory activity. Techniques that emphasize concentration and those that eschew it should be expected to have different EEG correlates. Techniques concentrating on specific psychological dispositions and/or cognitive attitudes and those ignoring them might well be expected to have different outside-of-meditation effects as well. And such differences are now widely reported in meditationrelated research. Until not too long ago, however, the results of meditation-related research were often misinterpreted. Even trained scientists doing literature reviews often still labored under the misconception that all meditation techniques are essentially the same, lumped results of research on different techniques together indiscriminately, and concluded from the jumble of conflicting results that "meditation" (understood generically) did not produce any significant results at all. However as the volume and sophistication of studies of meditation has grown, complimented by the increasing numbers of meta-analyses, this mistake has diminished significantly. It has become evident that meditation can often have significant effects, and as a result attention is in-

\footnotetext{
${ }^{4}$ Adapted from the Introduction to Shear (ed.), p. 9.

${ }^{5}$ Ibid., pp. 9-10.
} 
creasingly being given to distinguishing which procedures produce what kinds of results. ${ }^{6}$ These differences are considerable, and already falsify the uncritical perennialist idea that all meditation systems are essentially the same.

The mistaken idea that different forms of meditation are all essentially the same, however, is still all too common outside the scientific community. Prompted by the growing body of research, a wide variety of articles in all sorts of venues have popularized the idea that meditation can be beneficial in a variety of ways: reducing stress, enhancing overall health and well-being, and addressing a number of specific problems. This is a good thing, to the extent that it reflects the research accurately. But as it stands, it is also problematic. Well-meaning physicians, psychologists and other professionals, writing in venues likely to appear authoritative to the average reader, often lump techniques together incautiously, and imply and/or explicitly state that results reported from one technique can be expected from another one different from the first. And they often even go so far as to describe this second technique and recommend it as essentially the same as, or even identical to the one the research was conducted on, even when it clearly is not. ${ }^{7}$

\footnotetext{
${ }^{6}$ Compare, for example, the section on meditation in the American Heart Associations' recent study, "Beyond Medications and Diet: Alternative Approaches to Lowering Blood Pressure: A Scientific Statement From the American Heart Association" (Brook, et. al., 2013) pp. 4-7.

${ }^{7}$ Compare, the following from a recent Psychology Today blog, "Reading Between the (Head)Lines," (http://www.psychologytoday.com/blog/reading-between-the-headlines/201310). The author, a physician (whom as apparently quite well intentioned I prefer not to cite by name), reports here that According to a report published in the Journal of Alternative and Complementary Medicine a meta-analysis of TM analyzed 16 trials and 1,295 participants. The conclusion was that TM worked better in reducing severe anxiety than psychotherapy or other relaxation techniques. Medication was not addressed.
}

This is simple news reporting. But the author then adds,

The good news is that it's not magic and you do not need to take a class, although instructors are recommended. TM is a simple technique that can be easily learned and takes only 15 to 20 minutes twice a day.

Want to learn how to perform TM? Here's the ultra-simplified way to do it.

The author's implication is straightforward: Practice the method he describes and ("the good news") the anxiety-related results described in the research can be expected. The problem is that the his technique involves "concentrating" to block out. . . intrusive thoughts," but as anyone who has actually learned to practice the technique the research was conducted on knows, that doing this will actually stop it from working, as teachers of the technique repeatedly emphasize. The standard EEG research on concentration and TM practice furthermore reinforce this observation. For while the practice of TM is associated with $\alpha_{1}$ EEG, concentrating and "blocking out thoughts" are standard $(\gamma-\beta)$-associated, $\alpha$-blocking activities (compare Schomer, 2012, p. 936). Thus what the author is recommending is in fact very different from TM practice, both objectively as well as subjectively. Furthermore to my knowledge there is no evidence that what he describes has the anxiety-reducing effects described in the research cited. The point here is not whether the author recommends might or might not be useful, but that his uncautious assumption that different meditation techniques are essentially the same and can be expected to produce comparable results, is made without reference to, and in fact contradicted by, relevant research, and thus misleading to his readers. For so far as I know, despite what the author 
This of course is a misuse of research results, even if the two practices might at first glance seem similar. Results found to follow from one medication, for example, cannot simply be presumed to follow from another medication, even when the second one seems very similar. ${ }^{8}$ (Indeed claiming without specific supporting research that a given medication will gain the same results as those shown for another one is medically so inappropriate that it is typically illegal in the Western world.) The same logic holds for two similar-seeming meditation techniques.

This is a very real practical problem. People have different reasons for exploring meditation, and different meditation techniques can have very different kinds of results. ${ }^{9}$ This is why meditation traditions frequently have different techniques for different purposes. ${ }^{10}$ And when people under the misconception that meditation techniques are all pretty much the same try a technique neither associated with nor even intended to get the results they desire, disappointment and even discounting meditation in general is a natural result. This result, too, is often reflected in the media.

The remedy for all this is of course genuine, well-established knowledge. And for this it seems to me what we really need is a concerted, cross-tradition effort to determine which meditation techniques produce (i) what subjective experiences and states and (ii) what physiological, psychological and behavioral effects on (iii) what subpopulations over (iv) what time frames. The relevant knowledge is already emerging. Science naturally develops in this way. But working towards this end consciously can speed things up. If even a fraction of the testable claims meditation traditions around the world have made about types of experience and developmental potentials of human consciousness turn out to be true, consciousness studies and related fields can be expected to benefit greatly. Well-established, nuanced knowledge of which techniques produce what effects is also precisely what is needed to alleviate the practical, media-related difficulties discussed above, and enable people to determine which techniques might best serve their own particular needs and interests.

\footnotetext{
indicates, there is no evidence to suggest that the technique he describes is likely to produce the results described.

${ }^{8}$ Substituting a " $\mathrm{t}$ " for the "c" in "medication" in the sentence above should make this obvious. Is this note necessary?

${ }^{9}$ This, of course, is why meditation traditions often have different techniques for different purposes, e.g., settling into silence within (dhyana in Yoga, Vedanta/TM, shamata in Buddhist traditions.), gaining insight (samyama, vipassana, etc.), physical health, etc.

${ }^{10}$ Compare, for example, the use of shamata and vipassana in Buddhism, and dhyana and samyama in Yoga for settling into silence within and gaining insight, respectively, the myriads of techniques for physical health, etc.
} 
Such practical considerations, moreover, should not be ignored, even in a highly intellectual field such as consciousness studies. Ethics requires this. Research has for decades been reporting various positive psychological, physiological and behavioral effects in the direction of those widely claimed by meditation traditions. For many if not most researchers, this is the most interesting and important aspect of meditation research. And for most meditation traditions concern with practical, lived results is central, as exemplified in a parable attributed to Buddha:

If one has been shot by an arrow smeared with poison, he should let the surgeon remove it and clean the wound as soon as possible, rather than spending time first inquiring about the archers name, caste and status, the bow's shape and composition, the wood of the arrow, the fiber of the bowstring, the composition of the arrow-point first. If he did the latter, "he would simply die, and these things would still remain unknown to him." 11

For all the kinds of reasons discussed above, practical as well as intellectual, the task of sorting out which techniques produce what effects on what populations has seemed to me to be most important, and with this in mind my own work has focused increasingly on questions of sorting out similarities of and differences between meditation practices that might be useful to researchers. Two simple, but very different, typologies have emerged so far. The first categorizes meditation practices in terms of differences of what people actually do, phenomenologically speaking, while meditating. The second categorizes them in terms of differences of how the practices appear to relate to the spectra of effects produced.

The first typology directly reflects the phenomenology of what people actually do while meditating. Meditation researchers have often divided all meditation techniques into two types, "Focused Attention" (FA) and "Open monitoring" (OM). In Focused Attention, as the expression suggests, the meditator's intent is to keep his or her attention on a single object, whether his or her breath, a bodily part, a koan or paradox, or some other phenomenological object (as in the list described earlier), to the exclusion of all other experiences. In Open Monitoring the meditator's intent is to simply observe what passes through his (or her) awareness, without becoming involved with the contents of one's experience, and return to this monitoring if he notices that his attention is captured by a thought, feeling or other mental object. The work I was involved in added another category, Automatic Self-Transcending, to reflect practices in which there is no attempt to sustain any particular condition at all. Practices of this kind, once started, are reported

11 "Parable of the Arrow", Wikipedia, http://en.wikipedia.org/wiki/Parable_of_the_arrow, December 28, 2013. 
to automatically "transcend" their own activity and disappear, to be started up again later if appropriate. Identification of this category was motivated by the fact that (in contrast to both Focused Attention and Open Monitoring) it reflects what many meditators report they actually do in meditation, either because they were taught explicitly to meditate in this way (as in the case of $\mathrm{TM}$ ), or, in some instances, because they found themselves practicing other techniques (from Zen and Qigong, for example) in this way once they had become effortless and automatic through years of practice. ${ }^{12}$

This new tripartite typology, reflecting a more nuanced attention to the phenomenology of what people actually do while meditating, then turned out to be useful for EEG researchers. Different cognitive styles are naturally associated with different types of brain activity, and as I learned from neuroscientist Fred Travis, previous research suggested that the kinds of mental activities characteristic of Focused Attention, Open Monitoring and Automatic Self-Transcending were likely to be associated with $(\gamma \text { and } \beta)^{13}, \theta$, and $\alpha_{1}$ EEG bands, respectively. A literature search of EEG research on different kinds of meditation, conducted earlier by Travis, turned out to support this prediction. This in turn allowed the results of the literature search to be interpreted much more simply ${ }^{14}$ (Travis $\&$ Shear 2010). These kinds of results highlight the importance of attending to the specifics of the phenomenology of different meditation practices as carefully as we can, in the service both of interpreting prior studies and of generating typologies for new ones.

The tripartite typology above distinguishes meditation techniques in terms of the phenomenologies of what meditators actually do while meditating. The second typology mentioned earlier is quite different. It has two categories, "Practice-Makes-Perfect" and "State-Enlivening", to reflect two major ways techniques have been thought to produce their effects. "Practice-MakesPerfect" refers to techniques that intend to cultivate dispositions held to be of value by practicing

\footnotetext{
12 The categories of this typology are intended to distinguish techniques by phenomenology — what meditators actually do while meditating - regardless of how they may be referred to by name. As a result techniques referred to by a single name ("Zen", "Qigong", etc.) may at times be subsumed under more than one category, depending on phenomenology of the practice.

${ }^{13}$ Gamma often is used to include EEG from $20-50 \mathrm{~Hz}$. However, the frequency of 20-30 Hz is also referred to as " $\beta$ ". So to accommodate the results of studies that discriminate these two bands (or sub-bands) the locution " $(\gamma-\beta)$ " will be used from this point on.

14 (i) Focused Attention, (ii) Open Monitoring, and (iii) Automatic Self-Transcending were represented by practices from (i) Tibetan Buddhism, Diamond Way Buddhism, Qigong and Zen, (ii) Vipassana, Sahaja Yoga, Zen and Qigong, and (iii) TM and Qigong, respectively. References to the 17 studies of EEG and meditation the literature search located can be found in Travis \& Shear (2010).
} 
them in meditation. These include, for example, techniques that use extended focus on objects during meditation to enhance the ability to focus steadily in daily activity or enhance ones capacity for responding compassionately, and those that practice maintaining a non-evaluative, nonattached attitude toward whatever happens is experienced in meditation, in order to cultivate dispositions such as nonattached equanimity and objectivity in daily life. Many such techniques are both well known and widely practiced (e.g., traditional Vipassana and contemporary "Mindfulness"), and relevant psychological and physiological effects been reported in scientific studies. The latter include, for example, increased mass in relevant cortical regions correlated with years of meditation practice (Compare Lazar, et. al. 2005, pp. 1893-1897). ${ }^{15}$ The logic and effects of such practice-makes-perfect techniques thus seem quite straightforward: the mind/brain becomes better at doing things outside of meditation that it practices in an extended way during mediation.

Techniques in the second, "State-Enlivening" category are understood traditionally to produce major effects in a very different, if less obvious, way. Here the techniques are said to produce experience of unusual global states of consciousness, including in particular states devoid of phenomenological objects as discussed earlier, and it is these states, rather than the practices that led to them, that are thought to produce the desired after-meditation results. Many traditions (Zen, Tibetan Buddhism, Yoga, Vedanta, Taoism, etc.) often emphasize such techniques and states, and it is these kinds of techniques and states that caught my attention so many years ago. Zen koan work and the use of mantras in TM are prominent examples from the traditions I am now most familiar with. Both of these practices are said to produce experiences devoid of phenomenological objects, accompanied spontaneously by suspension of perceptible respiration. ${ }^{16}$ But the techniques themselves are strikingly different. Zen koan work involves intense concentration, and even mild concentration is characterized by $(\gamma-\beta)$ EEG (compare Shomer $\&$ da Silve (2012) p. 936). The use of mantras in TM eschews effort and concentration, and is characterized by $\alpha_{1}$ EEG. And $(\gamma-\beta)$-associated activity is so different from $\alpha$-associated activity that it said to be " $\alpha$-blocking." So the two techniques are clearly incompatible, both phenomenologically and electro-physiologically.

\footnotetext{
15 This study was conducted on long-term practitioners of "mindfulness," defined here as maintaining "a specific nonjudgmental awareness of present-moment stimuli without cognitive elaboration."

${ }^{16}$ For more on relationships between Zen, TM, contentless experiences and physiological correlates see Shear, J. \& Jevning, R. (1999).
} 
Furthermore, $(\gamma-\beta)$-associated, concentrative koan work is widely reported to enhance relaxed $\alpha$-associated mental activity in daily life, as well as, of course, $\gamma$-associated activity. Similarly, effortless $\alpha$-associated TM is widely reported to enhance $\gamma$-associated concentrative activities (e.g., mathematical performance.), as well as $\alpha$-associated ones. Both techniques, in other words, are reported to enhance both types of mental activities. This means that in both cases effects phenomenologically and electro-physiologically incompatible with the techniques practiced were produced. This of course is incompatible with a practice-makes-perfect account, and indicates that something other than practice-makes-perfect mechanisms must be at play.

What this "something" is remains to be seen. Perhaps, as the traditions the techniques come from hold in common, it is in fact a deep, apparently phenomenologically qualityless stratum of consciousness experienceable in meditation. Or perhaps the practices activate something more mundane that produces both the unusual experiences and the reported effects. This is an empirical question, relevant to many speculative topics in consciousness studies. But resolving it will require currently unavailable empirical theory and evidence. All such speculations aside, however, major differences between "Practice-Makes-Perfect" and "State-Enlivening" kinds of meditation techniques should by now be apparent.

We have so far seen something of how attention to often overlooked differences between meditation techniques can lead to new typologies better suited to extract information from existing studies, and presumably also of leading to new, useful types of comparative studies and meta-analyses. Let us now return to the topic of sameness of experiences these reflections began with. As noted earlier one of the things that first drew many people to the study of meditation was the congruence of reports of specific meditation-related experiences in widely different cultural and conceptual contexts. This congruence seemed to "filter out" these contexts as determinants of the nature of the experiences as reported, much as a scientific experiment might. This was not an actual scientific experiment, of course. All the data was purely anecdotal. A growing body of actual research however has now begun to examine relationships between these variables.

Consider, for example, the experience devoid of phenomenal objects referred to above. Traditional accounts of this experience often report respiratory suspension and unusual reduction of metabolic activity as correlates of this experience, and observation of respiratory suspension has often been by teachers in traditions as different as Chinese Zen (Chan) and Indian Sufism to 
identify episodes of the experience. ${ }^{17}$ Studies now provide evidence that, in laboratory settings, at least, reports of this experience are in fact highly correlated with suspension of perceptible respiration, along with unchanged levels of $\mathrm{CO} 2$ and $\mathrm{O} 2$ in the blood, and no compensatory breathing afterward. These findings both support and go beyond the traditional claims of respiratory suspension. The traditional use of respiratory suspension observed to identify episodes of this unique experience would thus appear to be on the right track Together with the traditional accounts, these findings also provide strong support for the idea that the use of terms such as "objectless" and "empty" reflects what people naturally experience while in this unusual physiological state, rather than cultural factors. For it does not seem at all plausible to think that so many different cultures and metaphysical contexts should simply serendipitously converge to produce these particular phenomenologically equivalent responses in correlation with this particular, highly unusual, unconscious physiological state.

Much more work, nevertheless, is needed here. We have been referring to "the" experience of objectless awareness as though only a single experience is being referred to. But traditional texts (in Buddhism and Yoga, for example) describe more than one experience that can reasonably be referred to as "objectless", and therefore "empty", as noted earlier. One such experience as described seems very much like experiencing nothingness itself, or even being nothingness itself. Nevertheless it is, as reported, a conscious mental event that, unlike unconsciousness, can actually be remembered. This experience, I would think, is the one that can most reasonably be referred to as completely empty. A second well known "objectless" experience can be described as consisting of nothing but one's point of view in the midst of vast emptiness. So in this case there is actually something in the experience, namely the bare subject-object phenomenological structure, even if the structure itself is devoid of objects. These experiences have important, sometimes overlapping, and sometimes very different, implications for issues in modern philosophy and psychology. But they are not usually distinguished carefully, if distinguished at all, even where one or the other may be discussed. Research specifically addressing these subtly different experiences and their respective correlates is needed. This will require, among other things, protocols identifying each experience in ordinary, tradition-independent language, both to facilitate cross-tradition research and to avoid tradition-associated biases. ${ }^{18}$ This task is not an

${ }^{17}$ Compare, for example, Chang, 1959 Llewellyn Vaughan-Lee, 1999).

${ }^{18} \mathrm{Cp}$ Zoran and me 
entirely easy one, as researchers have already found. Success however would allow researchers to determine whether respiratory suspension turns out to be a consistent identifier of either or both types of "objectless" experience. It is, I think, an important avenue to follow.

The logic of the above kinds of research can naturally be extended to other meditation-related experiences and/or putative correlates. Success here would of course have major implications for a wide range of topics in consciousness studies. ${ }^{19}$

There's a lot of work to be done, both here and in the more general, and, I think, most important task of determining which meditation techniques, produce what subjective experiences and objective effects. ${ }^{20}$ But it all appears to me to be well worth it. And as a Tibetan Lama remarked at a conference not long ago, "It's fun!"

\footnotetext{
${ }^{19}$ For an in principle discussion of some of these possible implications, see Shear, J., (2013).

${ }^{20}$ I would be remiss if I did not at least mention one additional area of meditation research, studies investigating relationships between meditation, the brain's "default mode network," and theories of self. This work is generating fascinating new approaches to theories of self, both Eastern and Western, and I suspect it will change the way we look at them significantly. See, for example, Travis, F., et al. (2010) and Brewer, J.A., et. al., (2014). For one suggested application, see pp. 49-50 of Shear, J. (2014).
} 


\section{References}

Brewer, J.A. et. al., (2014) "Meditation experience is associated with differences in default mode network activity and connectivity," Proceedings of the National Academy of Sciences, vol. 108 no. 50, pp. 20254-20259.

Brook, R.D., et. al. (2013) "A Scientific Statement From the American Heart Association Beyond Medications and Diet: Alternative Approaches to Lowering Blood Pressure," Hypertension, online April 22, 2013

Chang, C. C. (1959) The Practice of Meditation (New York: Perennial Library, Harper \& Row,)

Foreman, R. (ed.) (1990) The Problem of Pure Consciousness (Oxford University Press)

Katz, S. T., (ed.) (1978) "Language, Epistemology, and Mysticism," in Mysticism and Philosophical Analysis (New York: Oxford University Press) pp. 33, 59, 62, etc.

Sara W. Lazar, S. W. et. al. (2005) "Meditation experience is associated with increased cortical thickness", Neuroreport, Nov. 28, 2005, 16(17), pp. 1893-1897.

Schomer, D. L. \& da Silve, F. L. (2012) Niedermeyer's Electroencephalography: Basic Principles, Clinical Applications, and Related Fields (Google eBook: Lippincott Williams \& Wilkins) p. 936

Shear, J. (1981) "Maharishi, Plato and the TM-Sidhi Program on Innate Structures of Consciousness," Metaphilosophy, Vol. 12, No. 1

Shear, J. (1982) “The Universal Structures and Dynamics of Creativity,” J. of Creative Behavior, V. 16, N. 3,

Shear, J. (1983) "The Experience of Pure Consciousness: A New Perspective for Theories of Self," Metaphilosophy, Vol. 14, No. 1

Shear, J. (1990a) “The Philosopher, the Yogi, and Enlightenment: Plato's Symposium and Patanjali's Yoga Sutras," Darshana International (International Philosophical Quarterly), India, Vol. XXX, No. 1

Shear, J. (1990b) The Inner Dimension: Philosophy and the Experience of Consciousness (Peter Lang, 1990b). A new edition is currently in press (UK: Harmonia, 2014).

Shear, J. (1990c) "Mystical Experience, Hermeneutics, and Rationality," International Philosophical Quarterly, Vol. XXX, No. 4

Shear, J. (1994) "On Mystical Experiences as Support for the Perennial Philosophy," J. of the American Academy of Religion, Vol. 62, No. 2 
Shear, J. \& Jevning, R (1999) "Pure Consciousness: Scientific Exploration of Meditation Techniques," J. of Consciousness Studies, Vol. 6, No. 2-3, 1999, reprinted in Varela, F. \&. Shear, J. (editors) 1999.

Shear, J (2001) "Ethics and the Experience of Happiness" in Barnard, G. W. and Kirpal, J. J. (editors) Crossing Boundaries: Essays on the Ethical Status of Mysticism (Seven Bridges Press/Chatham House)

Shear, J (editor) (2006) The Experience of Meditation: Experts Introduce the Major Systems (Paragon House)

Shear, J., (2013) "Meditation as First-Person Methodology: Real Promise — and Problems," in Schmidt, S. and Walach, H. (editors) Meditation: Neuroscientific Approaches and Philosophical Explanations (Studies in Neuroscience, Consciousness and Spirituality (Springer).

Shear, J., (2014) "Converging on the Self: Western Philosophy, Eastern Meditation, Scientific Research," in Menon, S., Sinha, A. and Sreekantan, B.V. (editors) Interdisciplinary Perspectives on Consciousness and the Self (Springer).

Donald L. Schomer, Fernando Lopes da Silve (2012) Niedermeyer's Electroencephalography: Basic Principles, Clinical Applications, and Related Fields (Google eBook, Lippincott Williams \& Wilkins) p. 936

Travis, F. \& Shear, J. (2010) "Focused Attention, Open Monitoring and Automatic SelfTranscending: Categories to Organize Meditations from Vedic, Buddhist and Chinese Traditions," Consciousness and Cognition, Volume 19, Issue 4

Travis, F., et al. (2010) "A self-referential default brain state: Patterns of coherence, power, and eLORETA sources during eyes-closed rest and Transcendental Meditation practice," Cognitive Processing, vol. 11, no. 1, pp. 21-30.

Varela, F. \&. Shear, J. (editors) (1999) The View From Within: First-Person Approaches to the Study of Consciousness (Lawrence, KS: Imprint Academic)

Vaughan-Lee, L. (2006), "The Sufi Meditation of the Heart" in Shear, J. (editor) The Experience of Meditation (Paragon House) 